\title{
Hypersexuality in men with prolactinomas treated with dopamine agonists
}

\author{
Irina Bancos ${ }^{1} \cdot$ Todd B. Nippoldt ${ }^{1} \cdot$ Dana Erickson ${ }^{1}$
}

Received: 13 January 2017 / Accepted: 20 January 2017 / Published online: 4 February 2017

(C) Springer Science+Business Media New York 2017

Prolactinoma is the most common hypersecreting tumor of pituitary gland [1] and medical therapy with dopamine agonists (DAs) is recommended as first line intervention. DA therapy is effective in reversing hyperprolactinemia and its consequences-secondary hypogonadism and galactorrhea and may induce tumor shrinkage [2]. DA, such as cabergoline and bromocriptine, lead to prolactin normalization by acting through D2 receptors on the lactotroph cells. In many cases DA therapy is necessary for extended period of time, even life-long. While more common side effects of DAs such as headaches, dizziness and gastrointestinal disturbance are well known, a less recognized side effect of DA therapy is development of impulse control disorders (ICDs).

ICDs include hypersexuality, gambling, punding, compulsive shopping and binge eating and are thought to be mediated through dopamine receptors in the reward pathways of the mesolimbic system [3]. In a case-control study of 77 patients with DA treated prolactinomas and 70 patients with non-functioning pituitary tumors, prevalence of ICDs was reported to be 25 and $17 \%$, respectively [4]. However, striking differences were observed only for men in the DA treated- prolactinoma group who were almost 10 times more likely to develop an ICD when compared to men with non-functioning pituitary adenomas [4]. Moreover, authors noted a between-group significant difference only for hypersexuality which affected 10 patients (7 men) [4].

Irina Bancos

Bancos.Irina@mayo.edu

1 Division of Endocrinology, Metabolism and Nutrition, Mayo Clinic, Rochester, MN 55905, USA
In the current report, De Souza et al. describe findings in 8 men with DA treated prolactinomas identified from their clinics who developed hypersexuality. Authors suggest that a synergy between DA therapy and restoration of eugonadal state may contribute to development of hypersexuality and propose the term "dopa-testotoxicosis" to describe these occurrences [5]. In their case series, men developed hypersexuality within days to years after commencing DA therapy and this coincided with normalization of prolactin and improvement of testosterone concentrations. Interestingly, though hypersexuality was a predominant ICD, both Bancos et al. and De Souza et al. reported that a third of patients in their cohort (7/19 and 2/8, respectively) developed multiple ICDs $[4,5]$. The term "dopa-testotoxicosis" does not account for ICD development other than hypersexuality, or the development of hypersexuality in women. In addition, it is difficult to draw conclusions that a relative increase in testosterone concentrations (as none of the patients had supraphysiological testosterone concentrations) played a major contribution to developing hypersexuality, especially, given the design of both studies. Prospective studies are needed to reveal any temporal relationship to testosterone concentrations and to define any other predisposing factors to developing hypersexuality and other ICDs.

Based on the published case reports of hypersexuality reported in patients with DA treated prolactinomas, severity of presentation varies, but can be devastating to patients and their families. While some patients experience no or mild interference of symptoms with relationship and work, in others, symptoms can lead to loss of income, divorce, friends and family estrangement, promiscuity and legal troubles. Many times, since behavioral changes are not typical drug side effects, patients are not aware of possible causal relationship to DA therapy and may not volunteer the 
information on new symptom development to their physician.

It is reassuring to note that hypersexuality resolved either after DA reduction or cessation $[4,5]$.

Whether there is a DA dose or temporal relationship present, is unknown. It is unclear if there are predisposing factors to the development of ICD with DA therapy. Prospective observational trials are needed to determine a true prevalence and gender distribution of ICDs, risk factors for its development, and potential relationship to dose and duration of DA therapy.

What are the clinical implications with currently limited evidence? Endocrinologists need to recognize the possibility of ICD in relation to DA therapy, particularly its potential harmful effects and counsel patients appropriately.

Currently, at our center our approach is to:

1. Counsel the patient and family about the various ICDs as a potential side effect prior to initiation of therapy

2. If ICD occurs, we discontinue DA therapy to determine if the behavior resolves. If discontinuation does not resolve the ICD then we recommend a psychiatric evaluation. If ICD resolves after DA therapy discontinuation, we conclude that DA-induced ICD is likely. In this situation we consider and discuss the benefits of continued therapy for prolactinoma with the following options:

a. Replacement of hypogonadism without hyperprolactinemia reduction with biochemical and/or imaging monitoring of the pituitary tumor.

b. Reduction in dosing of initial DA agent or change to another DA agent with continued monitoring for redevelopment of ICD. c. Pituitary surgery or other tumor related therapy (such as stereotactic radiotherapy)

\section{Compliance with Ethical Standards}

Conflict of interest The authors declare that they have no competing interest.

Ethical approval This article does not contain any studies with human participants performed by any of the authors.

\section{References}

1. I. Bancos, N. Natt, M.H. Murad, V.M. Montori, Evidence-based endocrinology: illustrating its principles in the management of patients with pituitary incidentalomas. Best. Pract. Res. Clin. Endocrinol. Metab. 26, 9-19 (2012)

2. M.E. Molitch, Prolactinoma Management, eds. L.J. De Groot, G. Chrousos, K. Dungan, K.R. Feingold, A. Grossman, J.M. Hershman, C. Koch, M. Korbonits, R. McLachlan, M. New, J. Purnell, R. Rebar, F. Singer, A. Vinik, (Endotext, South Dartmouth, MA, 2000).

3. T.J. Moore, J. Glenmullen, D.R. Mattison, Reports of pathological gambling, hypersexuality, and compulsive shopping associated with dopamine receptor agonist drugs. JAMA Int. Med. 174, 1930-1933 (2014)

4. I. Bancos, M.R. Nannenga, J.M. Bostwick, M.H. Silber, D. Erickson, T.B. Nippoldt, Impulse control disorders in patients with dopamine agonist-treated prolactinomas and nonfunctioning pituitary adenomas: a case-control study. Clin. Endocrinol. 80, 863-868 (2014)

5. S.M. De Sousa, I.M. Chapman, H. Falhammar, D.J. Torpy, Dopatestotoxicosis: disruptive hypersexuality in hypogonadal men with prolactinomas treated with dopamine agonists. Endocrine doi:10. 1007/s12020-016-1088-1 (2016) 